\title{
Food insecurity and breastfeeding
}

\author{
Meta van den Heuvel MD PhD, Catherine Birken MD MSc
}

Cite as: CMAJ 2018 March 19;190:E310-1. doi: 10.1503/cmaj.180167

See related article at www.cmaj.ca/lookup/doi/10.1503/cmaj.170880

$\mathbf{F}$ ood insecurity - the unreliable access to sufficient quantities of affordable, nutritious food - is a serious public health concern in Canada. ${ }^{1}$ PROOF (Food Insecurity Policy Research) reported that $12 \%$ of Canadian households faced some level of food insecurity in 2014. ${ }^{2}$ In adults, food insecurity has been associated with poor physical and mental health, including chronic health conditions like diabetes and depression, and an increased rate of hospital admissions. ${ }^{3,4}$ Households with children have a greater risk for food insecurity; one in six Canadian children faced household food insecurity in $2014 .{ }^{2}$ Inadequate quality and quantity of food is associated with adverse outcomes in children. Children living in families that report food insecurity have reduced healthy food intake and lower academic outcomes, as well as higher rates of asthma, obesity, depression and anxiety. 2,5,6

In a linked study, Orr and colleagues ${ }^{7}$ report on the association of household food insecurity and breastfeeding initiation, the duration of exclusive breastfeeding and vitamin D supplementation of women who participated in the Canadian Community Health Survey (2005-2014).

Interestingly, the authors did not identify an association between food insecurity and the initiation of breastfeeding and the supplementation of vitamin D. A strength of the study is that the authors adjusted their analysis for important sociodemographic factors associated with breastfeeding, including education, partnership status, immigrant status, household income, Aboriginal identity and maternal health factors related to breastfeeding (mood disorders and diabetes). However, they did not include general ethnocultural identity in their analysis. Rates of breastfeeding initiation are strongly influenced by social and ethnocultural factors. ${ }^{8}$ In Canada, according to the 2009-2010 Community Health Survey, significantly more mothers who were of self-identified Asian (93.5\%) or black (93.9\%) background began breastfeeding than mothers who self-identified as white (86.7\%). ${ }^{9}$ To explore the association between breastfeeding initiation and food insecurity in more detail, future studies should explore ethnocultural factors.

The linked study did show an association between food insecurity and the duration of exclusive breastfeeding. Almost half of women in food-insecure households had ceased exclusive breastfeeding after two months, whereas half of the women in food-secure households breastfed exclusively for at least four months. Exclusive breastfeeding rates are low in Canada. In

\section{KEY POINTS}

- A better understanding about the biopsychosocial determinants of breastfeeding women who face food insecurity is necessary to develop effective interventions.

- Public policy should be extended to ensure that children receive optimal nutrition during sensitive periods of their early development.

- The government should plan a rigorous evaluation of the Canada Child Benefit program, with specific focus on maternal and child health outcomes among vulnerable recipients.

$2009-2010$, only $25.9 \%$ of mothers breastfed exclusively for the recommended six months. ${ }^{9}$ This rate was even lower in women in food-insecure households $(20.7 \%$ in the marginally foodinsecure group, $16.7 \%$ in the moderately insecure group and $15.7 \%$ in the severely food-insecure group). Surprisingly, only the women in the moderately food-insecure group had a significant risk of early cessation of exclusive breastfeeding (before six months) compared with mothers in food-secure households in the adjusted analysis. This may be explained by the relatively small number of women in the severely food-insecure group in the study, as acknowledged by the authors.

Another important confounder for both food insecurity and duration of breastfeeding is maternal employment. In the United States, early cessation of breastfeeding is associated with nonmanagerial employment and a lack of job flexibility. ${ }^{10}$ In the 2009-2010 Canadian Health Survey, 9\% of women identified the need to return to employment as a reason to stop breastfeeding. Therefore, an alternative explanation for the nonsignificant association between women in the marginally and severely foodinsecure groups and the duration of breastfeeding could also be explained by a difference in employment status. Other unmeasured sociobiological factors - such as comorbid medical or mental health problems, use of medications or maternal weight status - could also explain the association.

Despite these limitations, and those raised by the authors, the current study provides an important contribution to the literature on food insecurity and key maternal and child health outcomes. It is alarming that "women who can least afford to buy 
infant formula and whose babies can benefit most from the health-promoting qualities of breast milk are the least likely to breastfeed." 11,12 Many social, emotional and cultural barriers undermine breastfeeding, and for many women, exclusive breastfeeding is not feasible. ${ }^{11}$ However, the literature does not provide us with an answer about why women in food-insecure households in Canada stop exclusive breastfeeding early. A better understanding about the biopsychosocial determinants of breastfeeding in this vulnerable population is necessary to develop effective interventions.

Canada's policy relating to infant food security focuses mainly on promoting breastfeeding as the pathway to infant food security. Relying on promoting breastfeeding alone to address nutrition in vulnerable children is inadequate. ${ }^{11}$ In contrast to the US, where the Women, Infants and Children program provides food supplementation to infants and children, there is no national feeding program for infants and children in Canada. When Canadian mothers who report food insecurity require nutritional supplementation for their infants, they are left to rely on local food banks to obtain formula. The local policies of these food banks vary with respect to formula provisioning, likely resulting in differing access to appropriate nutrition for infants. ${ }^{11}$ Other options for nutritional supplementation to breastfeeding may include access to donor breast milk; however, this is limited in Canada. Intensifying public policy to ensure optimal nutrition during sensitive periods of early child development is essential to improve child health outcomes. ${ }^{12}$

Measures to increase household income comprise a promising strategy to promote food security. A recent randomized clinical trial showed that in the United Kingdom, financial incentives improved breastfeeding rates in areas with low breastfeeding prevalence. ${ }^{13}$ In Canada, the recent introduction of the nationwide Canada Child Benefit might have an important impact on household food insecurity. However, a rigorous evaluation of this income support program is needed, with specific focus on maternal and child health outcomes, to answer key questions; for example, "Will mothers who report food insecurity have equitable access to these benefits?" To improve outcomes for foodinsecure families, policy-makers must evaluate the Canada Child Benefit program by examining child and family health outcomes, and providers of child health, researchers and tax-payers must ensure that policy-makers do so.

\section{References}

1. Food insecurity. Vancouver: Dietitians of Canada; [updated 2016]. Available: www.dietitians.ca/dietitians-views/food-security/overview.aspx (accessed 2018 Jan. 5).

2. Tarasuk V, Mithcell A, Dachner N. Household food insecurity in Canada, 2014. Toronto: PROOF Food Insecurity Policy Research; 2016.

3. Vozoris NT, Tarasuk VS. Household food insufficiency is associated with poorer health. J Nutr 2003;133:120-6.

4. Jessiman-Perreault G, Mclntyre L. The household food insecurity gradient and potential reductions in adverse population mental health outcomes in Canadian adults. SSM - Pop Health 2017(3):464-72. Available: www.dx.doi.org/10.1016 /j.ssmph.2017.05.013 (accessed 2018 Jan. 5).

5. Kirkpatrick SI, Mclntyre LPM. Child hunger and long-term adverse consequences for health. Arch Pediatr Adolesc Med 2010;164:754-62.

6. Fuller A, Maguire JL, Carsley S, et al. Difficulty buying food, BMI, and eating habits in young children. Can J Public Health 2018;108:e497-e502.

7. Orr SK, Dachner N, Frank L, et al. Relation between household food insecurity and breastfeeding in Canada. CMAJ 2018;190:E312-9.

8. Balogun OO, O'Sullivian EJ, McFadden A, et al. Interventions for promoting the initiation of breastfeeding. Cochrane Database Syst Rev 2016;(11):CD001688.

9. Breastfeeding initiation in Canada: key statistics and graphics (2009-2010). Ottawa: Statistics Canada; [modified 2012]. Available: www.canada.ca/en/health-canada/ services/food-nutrition/food-nutrition-surveillance/health-nutrition-surveys/ canadian-community-health-survey-cchs/breastfeeding-initiation-canada-key -statistics-graphics-2009-2010-food-nutrition-surveillance-health-canada.html (accessed 2018 Jan. 5)

10. Guendelman S, Kosa JL, Pearl M, et al. Juggling work and breastfeeding: effects of maternity leave and occupational characteristics. Pediatrics 2009;123:e38-46.

11. Frank L. The breastfeeding paradox: a critique of policy related to infant food insecurity in Canada. Food Cult Soc 2015;18:107-29.

12. Venu I, Van Den Heuvel M, Wong JP, et al. The breastfeeding paradox: relevance for household food insecurity. Paediatr Child Health 2017;22:180-3.

13. Relton C, Strong M, Thomas KJ, et al. Effect of financial incentives on breastfeeding. JAMA Pediatr 2018;172:e174523.

\section{Competing interests: None declared.}

This article was solicited and has not been peer reviewed.

Affiliations: Division of Paediatric Medicine, Department of Paediatrics, The Hospital for Sick Children; Department of Paediatrics, Faculty of Medicine, University of Toronto, Toronto, Ont.

Contributors: Both authors contributed to the conception and design of the work, drafted the manuscript, revised it critically for important intellectual content, gave final approval of the version to be published and agreed to be accountable for all aspects of the work.

Correspondence to: Meta van den Heuvel, mathilda. vandenheuvel@sickkids.ca 